\title{
Optical black holes in moving dielectrics
}

\author{
V. A. De Lorenci* and R. Klippert ${ }^{\dagger}$ \\ Instituto de Ciências, Universidade Federal de Itajubá, Avenida BPS 1303 Pinheirinho, 37500-903 Itajubá, Minas Gerais, Brazil \\ Yu. N. Obukhov \\ Instituto de Física Teórica, Universidade Estadual Paulista, Rua Pamplona 145 Paulista, 01405-900 São Paulo, São Paulo, Brazil
}

(Received 23 August 2002; published 29 September 2003)

\begin{abstract}
We study the optical paths of the light rays propagating inside a nonlinear moving dielectric medium. For rapidly moving dielectrics we show the existence of a distinguished surface which resembles, as far as the light propagation is concerned, the event horizon of a black hole. Our analysis clarifies the physical conditions under which electromagnetic analogues of gravitational black holes can eventually be obtained in laboratory.
\end{abstract}

DOI: 10.1103/PhysRevD.68.061502

One of the most striking and well established results of general relativity theory is the prediction of a bounded region in space from where nothing can escape, not even light. Such an object, a black hole, arises as the spherically symmetric solution of Einstein equations, and its analysis on the basis of the semiclassical quantum field theory was considered to be fundamental for the understanding of quantum gravity. In particular, in the context of semiclassical gravity, Hawking suggested that black holes can evaporate [1]. Since the temperature of Hawking radiation is very low, astrophysical events are unlikely to give evidence for such a phenomenon.

During recent decades efforts have been put into the development of alternative physical models in which such a prediction could be experimentally verified under laboratory conditions. This class of models was called "analogue gravity" [2], and it deals with the propagation of sound waves in moving fluids [3-6] and with the electrodynamics of nonlinear media [7-9], among others [10-13].

The search for electromagnetic black holes represents an interesting topic in the broad area of analogue gravity, but the effective construction of such structures still remains an open problem. In a recent work [14] a certain progress has been achieved in this direction. In particular, it was shown that the birefringence effect occurring in moving nonlinear dielectrics can be described in terms of optical geometries (also known as electromagnetic effective geometries). Accordingly, the propagation of electromagnetic light rays is governed by optical metric tensors:

$$
\begin{aligned}
g_{-}^{\mu \nu}= & \eta^{\mu \nu}+(\epsilon \mu-1) u^{\mu} u^{\nu}-\mu u^{(\mu} e^{\nu) \alpha} F_{\alpha \beta} u^{\beta} \\
& -\frac{1}{\epsilon} u_{\alpha} e^{\alpha(\mu} F^{\nu) \beta} u_{\beta}, \\
g_{+}^{\mu \nu}= & \eta^{\mu \nu}+(\epsilon \mu-1) u^{\mu} u^{\nu} .
\end{aligned}
$$

Here $\eta^{\mu \nu}$ is the background (flat) spacetime metric, and the magnetic permeability $\mu$ is assumed to be a constant. The

\footnotetext{
*Electronic address: delorenci@unifei.edu.br

${ }^{\dagger}$ Electronic address: klippert@unifei.edu.br

$\ddagger$ On leave from the Moscow State University. Electronic address: yo@ift.unesp.br
}

PACS number(s): 04.20.-q, 42.15.Dp

derivative $e^{\mu \nu} \doteq \partial \epsilon / \partial F_{\mu \nu}$ of the dielectric permittivity $\epsilon$ with respect to the electromagnetic field measures the nonlinear effects. The motion of the medium is described by the velocity vector $u^{\mu}$ measured in units of the velocity of light in vacuum (i.e., $c=1$ except specified otherwise). The meaning of Eqs. (1), (2) is that the wave vectors $K_{\lambda}=\partial_{\lambda} \Sigma$ lie on the null geodesics of the above two optical metrics, depending on their polarization state. Here $\Sigma$ denotes the corresponding wave-front surface.

The aim of the present work is to show explicitly how the analogue event horizons can be built from the above metric structures. We analyze the propagation of two light rays described by the optical metrics (1), (2). Despite birefringence, the horizons for both polarization modes are shown to coincide. The spherical configuration is discussed, and we find an analogue event horizon resembling the Schwarzschild event horizon. Further, we study the cylindrically symmetric configuration as a possibly simpler structure to be built in a laboratory.

Let us now examine the light propagation inside a moving nonlinear dielectric matter. Consider at first the spherically symmetric case with the background metric $d s_{\eta}^{2}=d t^{2}-d r^{2}$ $-r^{2} d \theta^{2}-r^{2} \sin ^{2} \theta d \varphi^{2}$ in which the dielectric flows radially with the velocity $u^{\mu}=\gamma(1, \beta, 0,0)$, where $\gamma \doteq\left(1-\beta^{2}\right)^{-1 / 2}$. We assume a point charge fixed at the origin, and correspondingly the only nonzero component of the electromagnetic field is then $F^{01}=E_{r}$. Thus $e^{01} F_{01}=E(d \epsilon / d E) \doteq \epsilon^{\prime}$, with $E=\left|E_{r}\right|$. The optical metric (1) then reads

$$
\begin{aligned}
& g_{-}^{00}=\left(\epsilon+\epsilon^{\prime}\right) \gamma^{2}\left(\mu-\frac{\beta^{2}}{\epsilon}\right), \\
& g_{-}^{01}=\left(\epsilon+\epsilon^{\prime}\right) \gamma^{2} \beta\left(\mu-\frac{1}{\epsilon}\right), \\
& g_{-}^{11}=\left(\epsilon+\epsilon^{\prime}\right) \gamma^{2}\left(\beta^{2} \mu-\frac{1}{\epsilon}\right), \\
& g_{-}^{22}=\sin ^{2} \theta g_{-}^{33}=-\frac{1}{r^{2}} .
\end{aligned}
$$

Note that $g_{+}^{\mu \nu}=\left.g_{-}^{\mu \nu}\right|_{\epsilon^{\prime}=0}$. For spherically symmetric flows $\beta=\beta(r)$, the two metrics $g_{ \pm}^{\mu \nu}$ evidently admit the two Kill- 
ing vector fields $\xi=\{\partial / \partial t, \partial / \partial \varphi\}$. Written in components, $\xi_{a}^{\mu}=\delta_{a}^{\mu}$ with $a=0,3$. The light then propagates along the null geodesics with the tangent vectors $K^{\mu}=\dot{x}^{\mu}=(\dot{t}, \dot{r}, \dot{\theta}, \dot{\varphi})$ in such a way that $\xi_{0}^{\mu} K_{\mu}=K_{0}$ and $\xi_{3}^{\mu} K_{\mu}=K_{3}$ are constants along the corresponding optical path. Here the "dot" denotes the derivative with respect to the affine parameters $s$ along the optically null geodesic lines. For simplicity, we choose the radial propagation with $K_{2}=0=K_{3}$. The path of the light ray is then determined by $\dot{t}=\dot{r}(n \pm \beta) /(n \beta \pm 1)$. Here $n$ $=\sqrt{\mu \epsilon}$ coincides with the refraction index of the medium in the absence of external fields. The plus (minus) sign corresponds to the wave propagating from (to) the origin. The radial coordinate of the photon then changes in time as

$$
\frac{d r}{d t}=\frac{n \beta \pm 1}{n \pm \beta} .
$$

For the case of the matter flow directed outwards, i.e., $\beta$ $>0$, the incoming light (with $\dot{r}<0$ ) apparently "freezes" at the radius $r_{h}$ such that $\left.n \beta\right|_{r_{h}}=1$. The same happens for an outgoing light in the inwardly directed flow: the "freezing" occurs then at $\left.n \beta\right|_{r_{h}}=-1$. Both cases are compactly summarized in

$$
\left.n^{2} \beta^{2}\right|_{r_{h}}=1 .
$$

It is worthwhile to note that freezing is absent for the light propagating in the same direction as the moving dielectric medium.

There is a clear physical interpretation of the above result. Recall that $\beta=v / c$ for the 3-velocity $v$ of the matter flow, and $v_{c}=c / n$ is the velocity of light in a medium with refraction index $n$ in the absence of external fields. Then we can straightforwardly recast Eq. (7) into the form of the relativistic transformation of the velocity:

$$
\frac{d r}{d t}=\frac{v \pm v_{c}}{1 \pm v v_{c} / c^{2}} .
$$

The freezing of light takes place at a surface on which the velocity of matter $v$ becomes equal to the velocity of light $v_{c}$ in the medium. The geometrical interpretation is also clear: we find that the metric coefficient $g_{-}^{11}\left(r_{h}\right)=0$ vanishes at the radius $r_{h}$, see Eq. (5). The same conclusion is true also for the second optical metric, $g_{+}^{11}\left(r_{h}\right)=0$, i.e., both polarization modes (1) and (2) behave similarly. For the radial motion considered above, the stationary metrics given in the Eqs. (1), (2) can be rewritten into the static "black hole" form

$$
d s_{ \pm}^{2}=g_{00}^{ \pm} d \widetilde{t}^{2}-\frac{d \widetilde{r}^{2}}{g_{00}^{ \pm}}-r^{2} d \theta^{2}-r^{2} \sin ^{2} \theta d \varphi^{2},
$$

with $g_{00}^{-}=\gamma^{2}\left(1-n^{2} \beta^{2}\right) / \mu\left(\epsilon+\epsilon^{\prime}\right)$, by means of the coordinate transformations

$$
d \tilde{t}=d t+\frac{g_{ \pm}^{01}}{g_{ \pm}^{11}} d r
$$

$$
d \tilde{r}=d r \sqrt{\left(g_{ \pm}^{01}\right)^{2}-g_{ \pm}^{00} g_{ \pm}^{11}} .
$$

We note, for the case described here, that $g_{00}^{ \pm}$is proportional to $g_{ \pm}^{11}$.

From the above analysis, which generalizes the previous calculations [11], we find that either an ultrarelativistic motion of the matter or a highly refringent medium $[15,16]$, as it is found in Bose-Einstein condensates (see, however, a relevant discussion in Ref. [10]), is needed in order to display such a horizon structure. Highly refringent media are usually quite dispersive, and thus the effective horizon would occur in this case only for a narrow range of frequencies. A similar feature occurs also for sonic analogue models, where the horizon is defined only in a narrow range of low frequencies. A practical realization of the analogue black hole configuration requires that either $n=n(r)$ or $\beta=\beta(r)$, or even both.

Recently, it has been proposed that Hawking temperature is a purely kinematic effect that is generic to Lorentzian geometries containing event horizons [17], and thus dependent only on the effective metric structure. For the spherically symmetric solution the Hawking temperature $T$ of the analogue black hole is straightforwardly calculated for the metric (3)-(6) as

$$
T=\left|\frac{\hbar \gamma^{2} \beta n}{2 \pi k_{B}}\left(\frac{\partial \beta}{\partial r}+\frac{\beta \epsilon^{\prime}}{2 \epsilon E} \frac{\partial E}{\partial r}\right)\right|_{r_{h}} .
$$

Equation (13) shows that the nonlinearity may significantly contribute to this temperature. It should be noted, however, that the complete understanding of the physical meaning of the Hawking temperature associated with an analogue black hole is still an open question [10], and it can be settled only after a detailed analysis of radiation processes at the horizon. It should be stressed that the approximation of geometrical optics becomes unreliable for the discussion of modes whose wavelengths are comparable with the size of the horizon, as occurs in Hawking radiation processes. Furthermore, Hawking radiation from analogue black holes should also face the problem of the possible difference between signal velocity and phase velocity.

Experimentally though, it appears to be a rather difficult task to maintain a stationary spherically symmetric and inhomogeneous flow. In order to exhibit a more realistic configuration, we will now focus on the cylindrical symmetry. A particular case of such a configuration is the vortex matter flow which was discussed recently $[18,19]$. Let us consider a more general situation of a rotating dielectric body subjected to an electric field $E_{z}$ directed along the axis $z$ of rotation [20]. The background metric is then of the form $d s_{\eta}^{2}=d t^{2}$ $-d \rho^{2}-\rho^{2} d \varphi^{2}-d z^{2}$. The electromagnetic field has the only nonzero component $F^{03}=E_{z}$ and we denote $e^{03} F_{03}$ $=E(d \epsilon / d E) \doteq \epsilon^{\prime}$, where $E=\left|E_{z}\right|$. For the matter 4-velocity we have $u^{\mu}=\gamma(1, \beta, \omega, 0)$, with $\gamma=1 / \sqrt{1-\beta^{2}-\rho^{2} \omega^{2}}$, while $\omega$ and $\beta$ are arbitrary functions of the radial coordinate $\rho$. The optical geometry (1) now reads

$$
g_{-}^{00}=\gamma^{2}\left[\mu\left(\epsilon+\epsilon^{\prime}\right)-\beta^{2}-\rho^{2} \omega^{2}\right],
$$




$$
\begin{aligned}
& g_{-}^{02}=\frac{\omega}{\beta} g_{-}^{01}=\gamma^{2} \omega\left[\mu\left(\epsilon+\frac{\epsilon^{\prime}}{2}\right)-1\right], \\
& g_{-}^{11}=\gamma^{2}\left[\mu \epsilon \beta^{2}+\rho^{2} \omega^{2}-1\right], \\
& g_{-}^{12}=\gamma^{2} \beta \omega(\mu \epsilon-1), \\
& g_{-}^{22}=\gamma^{2}\left[\mu \epsilon \omega^{2}-\frac{1}{\rho^{2}}+\frac{\beta^{2}}{\rho^{2}}\right], \\
& g_{-}^{33}=-1-\frac{\epsilon^{\prime}}{\epsilon} \gamma^{2} .
\end{aligned}
$$

For the motion under consideration, the above metric components, Eqs. (14)-(19), depend only on the radial coordinate $\rho$. Then, for this geometry we have the three Killing vectors $\partial / \partial t, \partial / \partial \varphi, \partial / \partial z$. In components, $\xi_{a}^{\mu}=\delta_{a}^{\mu}$, with $a$ $=0,2,3$. The null geodesic equations $K^{\mu} \nabla_{\mu} K_{\lambda}=0$ for the wave vector $K_{\lambda}$ in the optical metric $g_{-}^{\mu \nu}$ are then more easily solved by using the fact that $\xi^{\mu} K_{\mu}=$ const for each Killing vector $\xi^{\mu}$. That is, the covariant components $K_{0}, K_{2}, K_{3}$ of the wave vectors $K^{\mu}=\dot{x}^{\mu}=(\dot{t}, \dot{\rho}, \dot{\varphi}, \dot{z})$ are constant along the corresponding geodesic lines. The remaining component $K_{1}$ is obtained from the null condition $g_{-}^{\mu \nu} K_{\mu} K_{\nu}=0$, and the geodesic equation for the radial coordinate reads

$$
\dot{\rho}= \pm \sqrt{\left(g_{-}^{10} K_{0}+g_{-}^{12} K_{2}\right)^{2}-g_{-}^{11}\left[2 g_{-}^{02} K_{0} K_{2}+g_{-}^{00}\left(K_{0}\right)^{2}+g_{-}^{22}\left(K_{2}\right)^{2}+g_{-}^{33}\left(K_{3}\right)^{2}\right]} \text {, }
$$

where the plus (minus) sign corresponds to a light ray propagating from (to) the symmetry axis $z$. Also, $\dot{z}=g_{-}^{33} K_{3}$ and

$$
\dot{t}=\frac{\left[g_{-}^{00} g_{-}^{11}-\left(g_{-}^{01}\right)^{2}\right] K_{0}+\left[g_{-}^{02} g_{-}^{11}-g_{-}^{01} g_{-}^{12}\right] K_{2}+g_{-}^{01} \dot{\rho}}{g_{-}^{11}},
$$

$$
\dot{\varphi}=\frac{\left[g_{-}^{02} g_{-}^{11}-g_{-}^{01} g_{-}^{12}\right] K_{0}+\left[g_{-}^{11} g_{-}^{22}-\left(g_{-}^{12}\right)^{2}\right] K_{2}+g_{-}^{12} \dot{\rho}}{g_{-}^{11}} .
$$

By specifying the dynamics of the matter, i.e., the functions $\beta$ and $\omega$, all the metric components $g_{-}^{\mu \nu}$ will be given func-
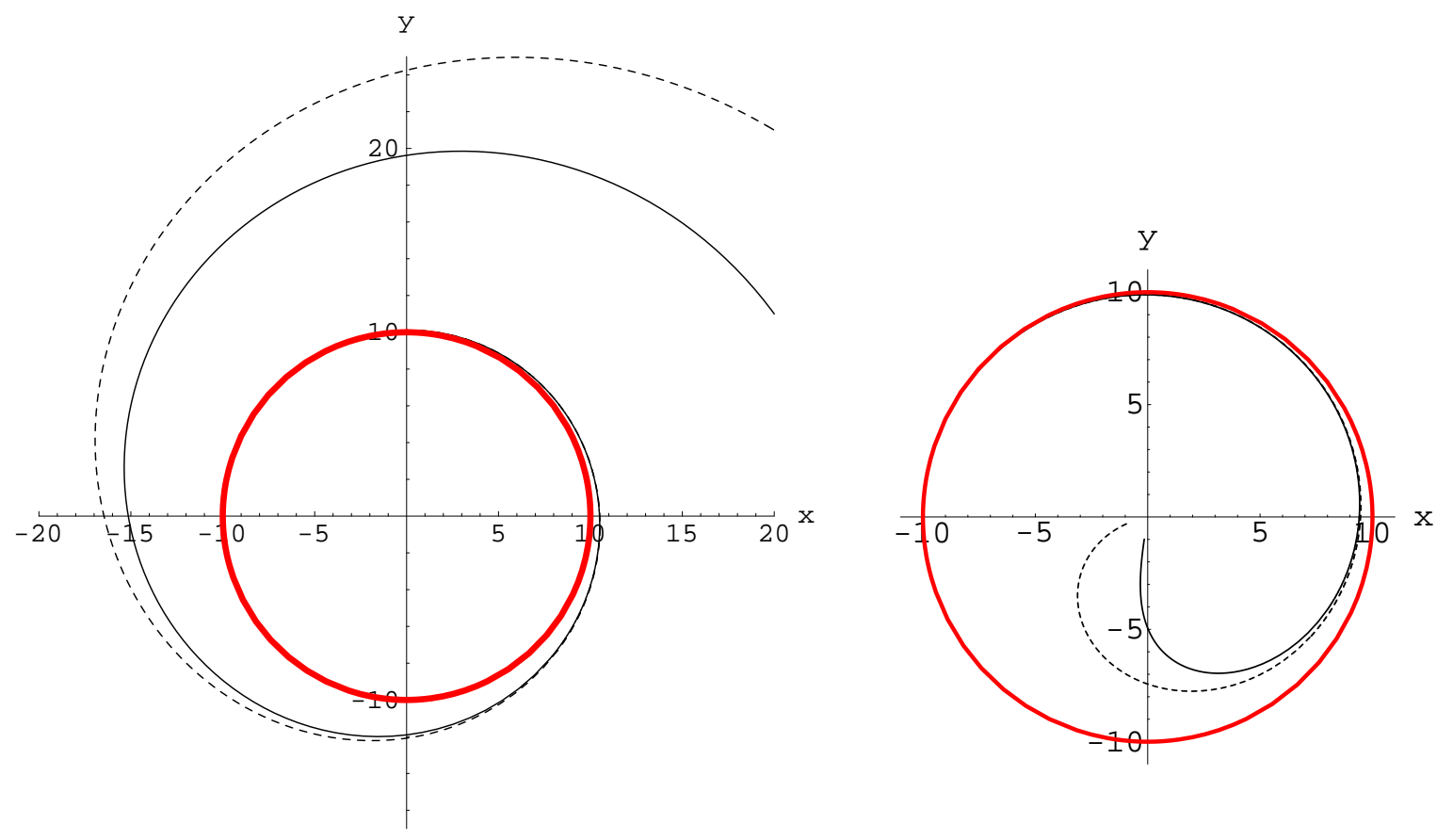

FIG. 1. The light paths for both polarization modes are shown for the cylindrical configuration. For simplicity, we assume in the plots a rigid rotation with $\omega=10^{-4}$, and the refraction index is $n=10^{3}$. The left/right figure corresponds to a dielectric fluid flowing outwards/ inwards, with radial velocity $\beta_{\text {out }}(\rho)=10^{-2} / \rho$ and $\beta_{\text {in }}(\rho)=-10^{-4} \rho$, respectively. This finite fluid configuration should hold only in the neigborhood of the region where the analogue horizon forms, since it would otherwise lead to a fluid with velocities larger than $c$. Besides, we take $\mu \epsilon^{\prime}=10^{10}$ for the extraordinary rays; the choice of a large value for $\epsilon^{\prime}$ is but illustrative, since the typical values would produce the rays too close to be distinguished from one another in the plots. The solid lines depict the ordinary rays, whereas the dashed lines represent the extraordinary rays. The thicker lines correspond to the analogue horizons in both cases. 
tions of $\rho$. Provided $\rho=\rho(s)$ is obtained from Eq. (20), the three other coordinates are then found by quadrature.

Let us now analyze the ordinary ray described by $g_{+}^{\mu \nu}$, which is obtained from the above computations when we set $\epsilon^{\prime}=0$. For simplicity, we also consider planar initial conditions for the light propagation such that $K_{2}=0=K_{3}$. Equations (20) and (21) then yield

$$
\frac{d \rho}{d t}=\frac{\gamma\left(n \beta+\sqrt{1-\rho^{2} \omega^{2}}\right)\left(n \beta-\sqrt{1-\rho^{2} \omega^{2}}\right)}{\left(n^{2}-1\right) \gamma \beta \mp \sqrt{n^{2}\left(1-\beta^{2}\right)-\rho^{2} \omega^{2}}} .
$$

As compared to the spherical case, Eq. (7), the solution of Eq. (23) displays the same qualitative behavior for the cylindrical configuration. The cylindrical horizon is located at $\rho$ $=\rho_{h}$ defined from

$$
\left.\left(n \beta \pm \sqrt{1-\rho^{2} \omega^{2}}\right)\right|_{\rho_{h}}=0 .
$$

Purely vortical motion of the fluid (with $\beta=0$ ) does not produce an analogue event horizon [12], since $\rho_{h}$ from Eq. (24) would lie beyond the limit of applicability of a rigid body in special relativity.

We note that $d \varphi / d \rho$ diverges at the surface defined by Eq. (24). As a result, the incoming/outgoing geodesic light rays spiral towards a horizon radius $\rho_{h}$, which can be explicitly demonstrated by the numerical integration of $d \varphi / d \rho$, see Fig. 1. Moreover, the condition of a horizon for the optical metric $g_{-}^{\mu \nu}$ is precisely given by Eq. (24). In terms of the optical metrics, Eqs. (14)-(19), the position of the horizon is defined by the equation $g_{ \pm}^{11}\left(\rho_{h}\right)=0$. In Fig. 1, we also depict the extraordinary ray which presents a qualitative behavior quite similar to its ordinary counterpart. Although the calcu- lations look somewhat more involved when the above initial conditions are not fulfilled, it can be shown that the horizon condition (24) remains unchanged. Therefore, we conclude that, for a given stationary flow configuration, the analogue horizon structure is of geometrical nature, because it depends neither on the initial conditions nor on the polarization of the propagating light rays.

Summarizing, Eq. (24) demonstrates how an experimental realization of a dielectric analogue horizon might be understood in terms of the parameters $n, \beta, \omega$ which describe the dielectric and kinematic properties of the medium. We expect that such horizons can be observed for stationary inhomogeneous kinematic configurations of the dielectric matter flow. The cylindrical configuration is favored due to the combined effect of both the refraction index $n$ and also of the vorticity $\omega$ of the medium which yields a smaller threshold for the radial velocity $\beta$. The horizon structures in both cases are shown to be independent of the presence of nonlinearities in the permittivity tensor.

As a final remark, it is worthwhile to mention that the present scheme may provide room also for the formation of sonic horizons (dumb holes [3]) for the cases in which ultrasonic velocities are achieved.

In the present paper, the study of light propagation in a nonlinear moving dielectric medium has revealed the existence of a surface which displays some of the properties of the event horizon of a black hole. We thus confirm the possibility of creating electromagnetic analogues of gravitational black holes in laboratory conditions.

This work was partially supported by the Brazilian research agencies CNPq, FAPEMIG, and FAPESP.
[1] S.W. Hawking, Commun. Math. Phys. 43, 199 (1975).

[2] In Analog models for general relativity, Rio de Janeiro, 2000, information available at the address http://www.cbpf.br/ bscg/ analog

[3] W.G. Unruh, Phys. Rev. Lett. 46, 1351 (1981).

[4] W.G. Unruh, Phys. Rev. D 51, 2827 (1995).

[5] M. Visser, Class. Quantum Grav. 15, 1767 (1998).

[6] L.J. Garay, J.R. Anglin, J.I. Cirac, and P. Zoller, Phys. Rev. Lett. 85, 4643 (2000).

[7] V.A. De Lorenci and R. Klippert, Phys. Rev. D 65, 064027 (2002).

[8] V.A. De Lorenci and M.A. Souza, Phys. Lett. B 512, 417 (2001).

[9] M. Novello and J.M. Salim, Phys. Rev. D 63, 083511 (2001).

[10] I. Brevik and G. Halnes, Phys. Rev. D 65, 024005 (2002); W.G. Unruh and R. Schutzhold, ibid. 68, 024008 (2003).

[11] R. Schützhold, G. Plunien, and G. Soff, Phys. Rev. Lett. 88, 061101 (2002).
[12] M. Visser, Phys. Rev. Lett. 85, 5252 (2000); U. Leonhardt and P. Piwnicki, ibid. 85, 5253 (2000).

[13] R. Schützhold and W.G. Unruh, Phys. Rev. D 66, 044019 (2002); C. Barceló, S. Liberati, and M. Visser, Class. Quantum Grav. 18, 1137 (2001).

[14] Yu.N. Obukhov and G.F. Rubilar, Phys. Rev. D 66, 024042 (2002).

[15] O. Kocharovskaya, Y. Rostovtsev, and M.O. Scully, Phys. Rev. Lett. 86, 628 (2001).

[16] D.F. Phillips, A. Fleischhauer, A. Mair, R.L. Walsworth, and M.D. Lukin, Phys. Rev. Lett. 86, 783 (2001).

[17] M. Visser, Phys. Rev. Lett. 80, 3436 (1998).

[18] U. Leonhardt and P. Piwnicki, Phys. Rev. A 60, 4301 (1999); Phys. Rev. Lett. 84, 822 (2000).

[19] M. Marklund, D. Anderson, F. Cattani, M. Lisak, and L. Lundgren, Am. J. Phys. 70, 680 (2002).

[20] Such electromagnetic field configuration corresponds to the experimental situation of a rotating condenser. 